\title{
Riccati Equations for Strongly Stabilizable Bounded Linear Systems*
}

\author{
Ruth F. Curtain and Job C. Oostveen \\ Department of Mathematics, University of Groningen, \\ P.O. Box 800, 9700 AV Groningen, The Netherlands. \\ Fax:+31 503633976. \\ E-mail: R.F.Curtain@math.rug.nl, J.C.Oostveen@math.rug.nl
}

April 14, 1997

\begin{abstract}
We obtain necessary and sufficient conditions for the existence of strongly stabilizing solutions to Riccati equations for infinite-dimensional systems that are strongly, but not exponentially stabilizable. We apply the theory to derive a Riccati criterion for the existence of J-spectral factorizations and illustrate it by proving the strict positive-real and bounded-real lemmas for a class of strongly stable systems with colocated actuators and sensors.
\end{abstract}

\section{Key words}

Riccati equations, strong stability, infinite-dimensional linear systems, Popov function, positive-real lemma, bounded-real lemma

\section{Introduction}

Until recently, results on the existence of solutions to Riccati equations assumed exponential stabilizability. In this paper, we address the existence and uniqueness of strongly stabilizing self-adjoint solutions to the following Riccati equation on a separable Hilbert space $Z$

$$
A^{*} X z+X A z-\left(B^{*} X+N C\right)^{*} R^{-1}\left(B^{*} X+N C\right) z+C^{*} Q C z=0
$$

for $z \in D(A)$. Throughout this paper we assume that:

A1. A generates a $C_{0}$-semigroup $T(t)$ on $Z$;

\footnotetext{
${ }^{*}$ This research was carried out while the authors were visiting the Center for Research in Scientific Computation, North Carolina State University from January to March, 1997.
} 
A2. $B \in \mathcal{L}(U, Z)$, where $U$ is a separable Hilbert space;

A3. $C \in \mathcal{L}(Z, Y)$, where $Y$ is a separable Hilbert space;

A4. $N \in \mathcal{L}(Y, U), Q=Q^{*} \in \mathcal{L}(Y), R=R^{*} \in \mathcal{L}(U), R^{-1} \in \mathcal{L}(U)$.

The approach we take makes use of spectral factorizations. Earlier papers using a spectral factorization approach to Riccati equations are Molinari [14] and Kučera [13] for the finite-dimensional case, and Callier and Winkin [4] and Weiss [25, 24] for the infinite-dimensional case, assuming exponential stabilizability.

More recently, there has been a spate of papers using a spectral factorization approach to solutions of Riccati equations for a much more general class of systems than the class considered here (see Staffans [17, 18, 19, 20, 21, 22] and Weiss and Weiss [26]). The generality of the class of well-posed linear systems lies in the fact that $B$ and $C$ (and other operators) are allowed to be very unbounded; in our case they are assumed to be bounded. The justification for a paper devoted to our special class of bounded systems lies in the following:

- the papers by Callier and Winkin [4] and Weiss [25, 24] impose exponential stabilizability, which is stronger than the strong stabilizability that we assume.

- Curtain and Zwart consider strongly stabilizable systems, but they consider only the standard LQ Riccati equation in [8].

- In $[18,19,20]$, Staffans allows for unbounded $B$ and $C$, but considers only the standard LQ Riccati equation.

- Weiss and Weiss [26] concerns stable systems with unbounded $B$ and $C$ operators, but they impose a coercivity condition, which is stronger than ours.

- Staffans [17] considers the same problem as in Weiss and Weiss [26], but for strongly stabilizable and detectable systems.

- Staffans [21] considers the same general Riccati equation as we do for a more general class of systems, but because of this he only obtains sufficient conditions. In fact, all the new papers on strongly stabilizable and detectable systems $[17,18,19,20,21,22,26]$ obtain only sufficient conditions for the existence of a solution to the Riccati equation. We obtain necessary and sufficient conditions.

So, although our class of systems is more restrictive in that $B$ and $C$ are assumed to be bounded, the type of Riccati equation is quite general and most importantly we obtain necessary and sufficient conditions for the existence and uniqueness of strongly stabilizing solutions. Our approach follows the thesis of Weiss [25] very closely (see also Weiss [24]), but it is also influenced by the recent paper by Weiss and Weiss [26] (which was influenced by [25]). 
The class of systems we consider has immediate applications to models used by engineers for large flexible space structures, namely dissipative systems with colocated actuators and sensors (see Balakrishnan [2], Joshi [12] and Section 8 in Slemrod [16]). In fact, this was the original motivation for this paper. It is the classic example of systems that are strongly stabilizable but never exponentially stabilizable. As a motivating example, we present the model of dynamic boundary control of a vibrating square elastic plate, considered by You in [27].

Example 1.1 Consider a square elastic plate with one clamped edge $\Gamma_{0}$ and two simply supported edges $\Gamma_{1}$ and $\Gamma_{3}$. The last edge, $\Gamma_{2}$, is subject to dynamical equations involving the inertial force, inertial torque, external control force $f_{1}(t, y)$ and external control torque $f_{2}(t, y)$. The latter two constitute the input $u(t)=$ $\operatorname{col}\left(f_{1}(t), f_{2}(t)\right)$.

Let $\Omega=[0,1] \times[0,1]$ and let $w(x, y, t)$ be the transverse vibrations of the plate, for $x \in \Omega$. Define $\Gamma_{0}=\{(x, y) \in \Omega: x=0\}, \Gamma_{1}=\{(x, y) \in \Omega: y=0\}$, $\Gamma_{2}=\{(x, y) \in \Omega: x=1\}, \Gamma_{3}=\{(x, y) \in \Omega: y=1\}$.

The following is a suitable model for the vibrating plate.

$$
\frac{\partial^{2} w}{\partial t^{2}}(x, y, t)+\Delta^{2} w(x, y, t)=0 \quad \text { for }(x, y) \in \Omega
$$

with initial conditions

$$
\begin{aligned}
& w(x, y, 0)=w_{0}, \\
& \frac{\partial w}{\partial t}(x, y, 0)=w_{1} \quad \text { for }(x, y) \in \Omega
\end{aligned}
$$

and boundary conditions

$$
\begin{aligned}
& w(0, y, t)=\frac{\partial w}{\partial x}(0, y, t)=0 \\
& w(x, 0, t)=\frac{\partial^{2} w}{\partial y^{2}}(x, 0, t)=0 \\
& w(x, 1, t)=\frac{\partial^{2} w}{\partial y^{2}}(x, 1, t)=0 \\
& \frac{\partial^{2} w}{\partial t^{2}}(1, y, t)=\frac{\partial^{3} w}{\partial x^{3}}(1, y, t)+(2-\sigma) \frac{\partial^{3} w}{\partial x \partial y^{2}}(1, y, t)+f_{1}(t, y) \\
& \frac{\partial^{3} w}{\partial t^{2} \partial x}(1, y, t)=-\frac{\partial^{2} w}{\partial x^{2}}(1, y, t)-\sigma \frac{\partial^{2} w}{\partial y^{2}}(1, y, t)+f_{2}(t, y) .
\end{aligned}
$$

If we measure the linear and angular velocities along $\Gamma_{2}$, we obtain the measurement

$$
q(t)=\left[\begin{array}{c}
\frac{\partial w}{\partial t}(t, 1, y) \\
\frac{\partial^{2} w}{\partial t \partial x}(t, 1, y)
\end{array}\right]
$$


To obtain a suitable first-order formulation, we introduce certain spaces and operators.

$$
\begin{aligned}
\mathcal{H}_{0}= & \mathbf{L}_{2}(\Omega) \times \mathbf{L}_{2}(0,1) \times \mathbf{L}_{2}(0,1), \text { with the inner product } \\
\langle\hat{w}, \hat{v}\rangle_{\mathcal{H}_{0}}= & \int_{\Omega} w(x, y) v(x, y) d x d y+\int_{0}^{1} w_{1}(y) v_{1}(y) d y+\int_{0}^{1} w_{2}(y) v_{2}(y) d y, \\
\mathcal{U}= & \mathbf{L}_{2}(0,1) \times \mathbf{L}_{2}(0,1), \\
D\left(A_{0}\right)= & \left\{\hat{w}=\operatorname{col}\left(w(x, y), w_{1}(y), w_{2}(y)\right) \in H^{4}(\Omega) \times \mathbf{L}_{2}(0,1) \times \mathbf{L}_{2}(0,1) \mid\right. \\
& w(0, y)=\frac{\partial w}{\partial x}(0, y)=0, w(1, y)=w_{1}(y), \frac{\partial w}{\partial x}(1, y)=w_{2}(y), \\
& \left.w(x, 0)=\frac{\partial^{2} w}{\partial y^{2}}(x, 0)=w(x, 1)=\frac{\partial^{2} w}{\partial y^{2}}(x, 1)=0\right\} .
\end{aligned}
$$

We define $A_{0}: D\left(A_{0}\right) \rightarrow \mathcal{H}_{0}$ and $B: \mathcal{U} \rightarrow \mathcal{H}_{0}$ by

$$
A_{0}=\left[\begin{array}{ccc}
\Delta^{2} & 0 & 0 \\
-\left.\left(\frac{\partial^{3}}{\partial x^{3}}+(2-\sigma) \frac{\partial^{3}}{\partial x \partial y^{2}}\right)\right|_{x=1} & 0 & 0 \\
\left.\left(\frac{\partial^{2}}{\partial x^{2}}+\sigma \frac{\partial^{2}}{\partial y^{2}}\right)\right|_{x=1} & 0 & 0
\end{array}\right], \quad B_{0}=\left[\begin{array}{cc}
0 & 0 \\
I & 0 \\
0 & I
\end{array}\right] .
$$

Next,

$$
\begin{aligned}
\mathcal{H}_{1} & =D\left(A_{0}^{\frac{1}{2}}\right), \text { with the inner product } \\
\langle\hat{w}, \hat{v}\rangle_{\mathcal{H}_{1}} & =\left\langle A_{0}^{\frac{1}{2}} \hat{w}, A_{0}^{\frac{1}{2}} \hat{v}\right\rangle_{\mathcal{H}_{0}}, \\
D\left(A_{1}\right) & =\left\{\hat{h} \in \mathcal{H}_{1}: \exists \hat{w} \in \mathcal{H}_{0} \text { such that }\langle\hat{h}, \hat{v}\rangle_{\mathcal{H}_{1}}=\langle\hat{h}, \hat{w}\rangle_{\mathcal{H}_{0}} \forall \hat{v} \in \mathcal{H}_{1}\right\}, \\
A_{1} \hat{h} & =\hat{w}, A_{1}: D\left(A_{1}\right) \subset \mathcal{H}_{1} \rightarrow \mathcal{H}_{0}, \\
\mathcal{H} & =\mathcal{H}_{0} \times \mathcal{H}_{1}, \\
D(A) & =D\left(A_{1}\right) \times \mathcal{H}_{1} .
\end{aligned}
$$

Finally, we define $A: D(A) \rightarrow \mathcal{H}$ and $B \in \mathcal{L}(\mathcal{U}, \mathcal{H})$ by

$$
A=\left[\begin{array}{cc}
0 & I \\
-A_{1} & 0
\end{array}\right], \quad B=\left[\begin{array}{c}
0 \\
B_{0}
\end{array}\right] .
$$

In You [27] it is shown that $A: D(A) \subset \mathcal{H} \rightarrow \mathcal{H}$ is a closed, densely defined skewadjoint operator with compact resolvent $\left(A^{-1} \in \mathcal{L}(\mathcal{H})\right.$ is compact). A generates a unitary $C_{0^{-}}$group of operators $T(t)$ on $\mathcal{H}$. The system (2) with boundary conditions can be reformulated as

$$
\begin{aligned}
\frac{d z}{d t}(t) & =A z(t)+B u(t) \\
y(t) & =B^{*} z(t)
\end{aligned}
$$


for $z(t) \in \mathcal{H}$, with $u, y \in \mathbf{L}_{2}(0,1) \times \mathbf{L}_{2}(0,1)$ and $B \in \mathcal{L}(U, H)$.

In the same paper it is proven that $A-B B^{*}$ generates a strongly stable contraction semigroup $T_{B}(t)$. Since $A$ has compact resolvent, this implies that $T_{B}^{*}(t)$ is also strongly stable. He also shows that $\Sigma(A, B,-)$ is approximately controllable by appealing to results in Benchimol [3]. Here, we prove approximate controllability and observability by appealing to properties of Lyapunov equations from Hansen and Weiss [11] (See also Curtain and Zwart [7], Theorem 4.1.23). It is clear that the following Lyapunov equations have the solution $\frac{1}{2} I$

$$
\begin{aligned}
& \left(A-B B^{*}\right)^{*} L_{C} z+L_{C}\left(A-B B^{*}\right) z=-B B^{*} z, \quad z \in D(A), \\
& \left(A-B B^{*}\right) L_{B} z+L_{B}\left(A-B B^{*}\right)^{*} z=-B B^{*} z, \quad z \in D\left(A^{*}\right) .
\end{aligned}
$$

Since $T_{B}^{*}(t)$ and $T_{B}(t)$ are strongly stable, $L_{C}=\frac{1}{2} I=L_{B}$ are the unique solutions of (1.1) and (1.1), respectively. From the results in Section 3.7 of Hansen and Weiss [11]. we conclude that $\Sigma\left(A-B B^{*}, B, B^{*}\right)$ have identical controllability and observability gramians $L_{C}=\frac{1}{2} I=L_{B}$. As in Curtain and Zwart [7], Theorem 4.1.22, this shows that $\Sigma\left(A-B B^{*}, B, B^{*}\right)$ is both approximately controllable and observable. Since these properties are invariant under feedback, this implies that $\Sigma\left(A, B, B^{*}\right)$ is both approximately controllable and observable.

The preceding example has a very special state-space structure, namely, $\Sigma\left(A, B, B^{*}\right)$, where $A$ is dissipative and $B$ is a bounded operator. The duality between the input and output operators, $C=B^{*}$, is usually termed "colocated" in the literature, because the input and output are implemented at the same location. It is known that if $A$ has compact resolvent and either $\Sigma(A, B,-)$ is approximately controllable or $\Sigma\left(A,-, B^{*}\right)$ is approximately observable, then $A-B B^{*}$ generates a strongly stable $C_{0}$-semigroup (see Benchimol [3]). In fact, this is a common way of stabilizing distributed parameter systems of hyperbolic type. Another example modeling a flexible beam, but of the same mathematical structure can be found in Slemrod [16].

While our theory is directly applicable to the $\Sigma\left(A, B, B^{*}\right)$ class, it is more naturally formulated in a more general setting, using the well-posed system theory approach from Weiss [23].

In section 2, we give the definitions and results needed to prove our result. For completeness, we include proofs of two technical lemmas, that can be found in Weiss and Weiss [26] and in the earlier papers of Staffans [17, 18, 19, 20, 21, 22]; We have followed the notation in Weiss and Weiss [26]. In section 3, we prove the existence and uniqueness of a strongly stabilizing solution to the Riccati equation for stable systems. As a special case of this, we present a Riccati criterion for Jspectral factorizations. In section 4, we extend our results to the unstable case. We illustrate the usefulness of these results by applying them to obtain versions of the positive- and bounded-real lemma for dissipative systems with colocated actuators and sensors (the $\Sigma\left(A, B, B^{*}\right)$ class). Applications to $\mathbf{H}_{\infty}$-control design for these systems will be investigated in a future paper. 


\section{Preliminaries}

In this section we give definitions and results, which we need to prove the main results. To start with, we recall the definitions of some function spaces, which we will use throughout this paper.

Definition 2.1 Let $Z_{1}, Z_{2}$ be Hilbert spaces, $B$ a Banach space and $\Omega \subset \mathbb{C}$.

- $\mathbf{L}_{2}\left(\Omega, Z_{1}\right)$ is the class of Lebesgue-measurable, square integrable, $Z_{1}$-valued functions on $\Omega$.

- $\mathbf{H}_{2}\left(Z_{1}\right)$ is the class of holomorphic, square-integrable, $Z_{1}$-valued functions on the open right half-plane.

- $\mathbf{H}_{\infty}(B)$ is the class of bounded, holomorphic, B-valued functions on the open right half-plane.

- $\mathbf{L}_{\infty}\left(\mathcal{L}\left(Z_{1}, Z_{2}\right)\right)$ is the class of essentially bounded, weakly Lebesgue measurable, $\left.\mathcal{L}\left(Z_{1}, Z_{2}\right)\right)$-valued functions on the imaginary axis.

We have the following result for these function spaces.

Lemma 2.2 Let $U, Y$ be separable Hilbert spaces, and $F:\{s \in \mathbb{C} \mid \operatorname{Re}(s)>0\} \rightarrow$ $\mathcal{L}(U, Y)$. If $F(j \omega) \in \mathbf{L}_{\infty}(\mathcal{L}(U, Y))$ and $F(s) u \in \mathbf{H}_{2}(Y)$ for all $u \in U$, then $F(s) \in$ $\mathbf{H}_{\infty}(\mathcal{L}(U, Y))$.

Proof. $F(s)$ is analytic for $\operatorname{Re}(s)>0$, because $F(s) u \in \mathbf{H}_{2}(Y)$, for all $u \in U$. Let $\|F\|_{L_{\infty}}=M$ and let $s=x+i y$, with $x>0$. It is well known (see e.g. Theorem 11.2 in Duren [10]) that any function in $\mathbf{H}_{2}$ admits a Poisson integral representation. In our case:

$$
F(s) u=\frac{1}{2 \pi} \int_{-\infty}^{\infty} \frac{x}{x^{2}+(y-\omega)^{2}} F(j \omega) u d \omega .
$$

Hence, for every $s$ in the open right half-plane,

$$
\begin{aligned}
\|F(s) u\|_{Y} & \leq M\|u\|_{U} \frac{1}{2 \pi} \int_{-\infty}^{\infty} \frac{x}{x^{2}+(y-\omega)^{2}} d \omega \\
& =\frac{M}{2}\|u\|_{U} .
\end{aligned}
$$

Thus, we have that $F(s)$ is uniformly bounded and analytic on $\operatorname{Re}(s)>0$, i.e. $F \in \mathbf{H}_{\infty}(\mathcal{L}(U, Y))$.

Corollary 2.3 Let $U, Y$ be separable Hilbert spaces, and $F:\{s \in \mathbb{C} \mid \operatorname{Re}(s)>0\} \rightarrow$ $\mathcal{L}(U, Y) . F^{\sim}(s):=F^{*}(-s)$. If $F(j \omega) \in \mathrm{L}_{\infty}(\mathcal{L}(U, Y))$ and $F^{\sim}(-s) y \in \mathbf{H}_{2}(U)$ for all $y \in Y$, then $F(s) \in \mathbf{H}_{\infty}(\mathcal{L}(U, Y))$. 
Proof. $\quad F(s) \in \mathbf{L}_{\infty}(\mathcal{L}(U, Y))$ if and only if $F^{\sim}(-s) \in \mathbf{L}_{\infty}(\mathcal{L}(Y, U))$. Thus, Lemma 2.2 implies that $F^{\sim}(-s) \in \mathbf{H}_{\infty}(\mathcal{L}(Y, U))$. This, in turn, is equivalent with $F(s) \in \mathbf{H}_{\infty}(\mathcal{L}(U, Y))$.

For our main result, we also need some notions and results from the theory of well-posed linear systems and regular linear systems. Details and further references can be found in Weiss [23]. For any Hilbert space $W, S_{\tau}$ is the right shift on $\mathbf{L}_{2}(0, \infty ; W)$, defined by

$$
\left(S_{\tau} w\right)(t)= \begin{cases}0 & 0 \leq t<\tau \\ w(t-\tau) & t \geq \tau\end{cases}
$$

An operator $\mathbb{F}$ on $\mathbf{L}_{2}(0, \infty ; W)$ is called shift-invariant if $\mathbb{F} S_{\tau}=S_{\tau} \mathbb{F} . \quad P_{\tau}$ denotes the projection of $\mathrm{L}_{2}(0, \infty ; W)$ onto $\mathrm{L}_{2}(0, \tau ; W)$ by truncation, defined for $w \in \mathrm{L}_{2}(0, \infty ; W)$ by

$$
\left(P_{\tau} w\right)(t)= \begin{cases}w(t) & 0 \leq t<\tau \\ 0 & t \geq \tau\end{cases}
$$

For $w_{1}, w_{2} \in \mathbf{L}_{2}(0, \infty ; W)$ and $\tau \geq 0$, the $\tau$-concatenation of $w_{1}$ and $w_{2}$, denoted $w_{1} \diamond w_{\tau}$ is defined by

$$
\left(w_{1} \diamond_{\tau} w_{2}\right)(t)= \begin{cases}w_{1}(t) & 0 \leq t<\tau \\ w_{2}(t-\tau) & t \geq \tau\end{cases}
$$

We now define well-posed linear systems for the Hilbert spaces $Z, U, Y$.

Definition 2.4 $A$ well-posed linear system on $U, Z$ and $Y$ is a quadruple $\Sigma=$ $(\mathbf{T}, \boldsymbol{\Phi}, \Psi, \mathbf{F})$, where

1. $\mathbf{T}=\{T(t)\}_{t \geq 0}$, where $T(t)$ is a strongly continuous semigroup of bounded linear operators on $Z$

2. $\boldsymbol{\Phi}=\left\{\Phi_{t}\right\}_{t \geq 0}$ is a family of bounded linear operators from $\mathbf{L}_{2}(0, \infty ; U)$ to $Z$ such that

$$
\Phi_{\tau+t}(u \underset{\tau}{u} v)=T(t) \Phi_{t} u+\Phi_{t} v
$$

for any $u, v \in \mathbf{L}_{2}(0, \infty ; U)$ and any $\tau, t \geq 0$,

3. $\Psi=\left\{\Psi_{t}\right\}_{t \geq 0}$ is a family of bounded linear operators from $Z$ to $\mathbf{L}_{2}(0, \infty ; Y)$ such that

$$
\Psi_{\tau+t} x=\Psi_{\tau} x \diamond \underset{\tau}{\diamond} \Psi_{t} T(\tau) x,
$$

for any $x \in Z$ and any $\tau, t \geq 0$, and $\Psi_{0}=0$, 
4. $\mathbf{F}=\left\{\mathbb{F}_{t}\right\}_{t \geq 0}$ is a family of bounded linear operators from $\mathbf{L}_{2}(0, \infty ; U)$ to $\mathbf{L}_{2}(0, \infty ; Y)$ such that

$$
\mathbb{F}_{\tau+t}(u \diamond v)=\mathbb{F}_{\tau} u \diamond \underset{\tau}{\sim}\left(\Psi_{t} \Phi_{\tau} u+\mathbb{F}_{t} v\right)
$$

for any $u, v \in \mathbf{L}_{2}(0, \infty ; U)$ and any $\tau, t \geq 0$, and $\mathbb{F}_{0}=0$.

The four families of operators, defined above relate the input function $u$, the output function $y$, the state at time $t, x(t)$ and the initial state $x(0)$ of the system on any finite interval $[0, t)$ in the following way:

$$
\left(\begin{array}{c}
x(t) \\
P_{t} y
\end{array}\right)=\left(\begin{array}{cc}
T(t) & \Phi_{t} \\
\Psi_{t} & \mathbb{F}_{t}
\end{array}\right)\left(\begin{array}{c}
x(0) \\
P_{t} u
\end{array}\right)
$$

Both $\Psi_{t}$ and $\mathbb{F}_{t}$ have a limit as $t \rightarrow \infty$, where the convergence is in the Frechet space $\mathbf{L}_{2}^{\text {loc }}(0, \infty ; Y)$. These limits are denoted $\Psi$ (the extended output map) and $\mathbb{F}$ (the extended input-output map), respectively. Note that we use a boldface letter for the families of operators in Definition 2.4 and a normal letter for the corresponding extended operator. It can be checked that $\mathbb{F}$ is a shift-invariant operator (i.e. $S_{\tau} \mathbb{F}=$ $\mathbb{F} S_{\tau}$ ). It determines a function $G(s)$ which is called the transfer function of the system.

To every well-posed linear system $\Sigma$ we can associate a triple of operators $(A, B, C)$ such that for $t \geq 0$

$$
\begin{aligned}
& \Phi_{t} u=\int_{0}^{t} T(t-s) B u(s) d s, \\
& (\Psi x)(t)=C T(t) x, \text { for } x \in Z_{1} \subset Z,
\end{aligned}
$$

where $A$ is the generator of the semigroup $T(t)$, and $B$ and $C$ are (possibly unbounded) operators from $U$ to $Z$ and $Z$ to $Y$, respectively. If $C$ is bounded, then $Z_{1}=Z$. A well-posed linear system is called regular if the transfer function $G(s)$ has a strong limit as $s \rightarrow \infty$ along the real axis. A sufficient condition for a well-posed linear system to be regular is that the input operator $B$ be bounded. A regular linear system is generated by a quadruple $(A, B, C, D)$ of operators, such that $(3)$ and (4) hold and furthermore,

$$
(\mathbb{F} u)(t)=C_{L} \int_{0}^{\infty} T(t-s) B u(s) d s+D u(t),
$$

where $D u=\lim _{s \rightarrow \infty} G(s) u$ and $C_{L}$ is an extension of $C$ to a domain which includes $T(t) x$ for every $x \in Z$ and almost every $t$. If $C$ is bounded, then $C_{L}=C$. $(A, B, C, D)$ are called the generating operators of $\Sigma$. 
Finally, we define the operator

$$
\tilde{\Phi}_{\tau}=\int_{0}^{\tau} T(s) B u(s) d s
$$

Clearly, $\left\|\Phi_{\tau}\right\|=\left\|\tilde{\Phi}_{\tau}\right\|$. If $T(t)$ is exponentially stable, $\tilde{\Phi}_{\tau}$ has a limit $\tilde{\Phi} \in \mathcal{L}\left(\mathbf{L}_{2}(0, \infty ; U), Z\right)$ given by

$$
\tilde{\Phi} u=\lim _{\tau \rightarrow \infty} \tilde{\Phi}_{\tau} u
$$

This limit can also exist if $T(t)$ is not exponentially stable; in such a case, we call $B$ an infinite-time admissible input operator for $T(\cdot)$ and the operator $\tilde{\Phi}$ the extended input map. Analogously, $C$ is called an infinite-time admissible observation operator for $T(\cdot)$ if the extended output map $\Psi \in \mathcal{L}\left(Z, \mathrm{~L}_{2}(0, \infty ; Y)\right)$.

In our applications, $B$ and $C$ will always be bounded. To distinguish this special case from the general case we use the terminology bounded linear system $\Sigma(A, B, C, D)$ to mean the well-posed linear system $\Sigma$ with generating operators ( $A, B, C, D$ ), where $B, C$ and $D$ are bounded. Note that such a system is always regular and has the transfer function $G(s)=D+C(s I-A)^{-1} B$ and

$$
(\mathbb{F} u)(t)=\int_{0}^{\infty} C T(t-s) B u(s) d s+D u(t) .
$$

Moreover the dual system $\Sigma^{d}=\Sigma\left(A^{*}, C^{*}, B^{*}, D^{*}\right)$ can be defined in an obvious way with $A^{*}$ the generator of the $C_{0}$-semigroup $T^{*}(\cdot)$ on $Z$. It is straightforward to show that $C$ is an infinite-time admissible observation operator for $T(\cdot)$ if and only if $C^{*}$ is an infinite-time admissible control operator for $T^{*}(\cdot)$. Moreover, for any $w \in \mathbf{L}_{2}(0, \infty ; Y)$ with $\dot{w} \in \mathbf{L}_{2}(0, \infty ; Y)$ there holds

$$
\Psi^{*} w=\lim _{\tau \rightarrow \infty} \int_{0}^{\tau} T^{*}(s) C^{*} w(s) d s
$$

and

$$
\begin{aligned}
\left(\mathbb{F}^{*} w\right)(t) & =\left(B^{*} \Psi^{*} S_{t}^{*} w\right)(t)+D^{*} w(t) \\
& =B^{*} \lim _{\tau \rightarrow \infty} \int_{t}^{\tau} T^{*}(s-t) C^{*} w(s) d s+D^{*} w(t)
\end{aligned}
$$

for all $t \geq 0$.

For more general duality results for well-posed linear systems see Weiss and Weiss [26], section 6. The following result that was proven in Theorem 11.1 of the same paper (see also Lemma 4.10 of Staffans [20]) is not so obvious, even for bounded linear systems. Therefore, we include a proof for this special case.

Lemma 2.5 Let $T(\cdot)$ be a $C_{0}$-semigroup and let $\Psi$ be an extended output map for $T(\cdot)$. 
1. If $\overline{\mathbb{F}} \in \mathcal{L}\left(\mathbf{L}_{2}(0, \infty ; U), \mathbf{L}_{2}(0, \infty ; Y)\right)$ is shift invariant, then $\Psi^{n e w}=\overline{\mathbb{F}}^{*} \Psi$ is an extended output map for $T(\cdot)$;

2. Let $\bar{\Sigma}=(\overline{\mathbf{T}}, \overline{\mathbf{\Phi}}, \bar{\Psi}, \overline{\mathbf{F}})$ be a bounded linear system with generating operators $(\bar{A}, \bar{B}, \bar{C}, \bar{D})$ and suppose that $\bar{C}$ is an infinite-time admissible observation operator for $\bar{T}(\cdot)$. Then, $\Psi^{\text {new }}=\overline{\mathbb{H}}^{*} \Psi=C^{\text {new }} T(\cdot)$ is an extended output map for $T(\cdot)$, where

$$
C^{n e w} x_{0}=\bar{B}^{\star} V x_{0}+\bar{D}^{*} C x_{0},
$$

and $V$ satisfies

$$
V z=\bar{\Psi}^{*} \Psi z=\lim _{\tau \rightarrow \infty} \int_{0}^{\tau} \bar{T}^{*}(t) \bar{C}^{*} C T(t) z d t
$$

Proof. (1). We need to show that $\Psi^{\text {new }}$ satisfies Definition 2.4(iii), which reduces to showing that

$$
S_{\tau}^{*} \Psi^{n e w} x_{0}=\Psi^{n e w} T(\tau) x_{0} \quad \text { for } x_{0} \in Z .
$$

This follows from

$$
\begin{aligned}
S_{\tau}^{*} \Psi^{n e w} & =S_{\tau}^{*} \overline{\mathbb{H}}^{*} \Psi \\
& =\overline{\mathbb{F}^{*}} S_{\tau}^{*} \Psi \text { since } \overline{\mathbb{H}^{*}} \text { is shift-invariant } \\
& =\overline{\mathbb{F}}^{*} \Psi T(\tau) \\
& =\Psi^{n e w} T(\tau) .
\end{aligned}
$$

(2). From (4) it follows that

$$
C^{n e w} x_{0}=\left(\Psi^{n e w} x_{0}\right)(0)=\left(\overline{\mathbb{F}}^{*} \Psi x_{0}\right)(0) .
$$

Taking now $x_{0} \in D(A)$, we see that $w=\Psi x_{0}$ and $\dot{w}=\Psi A x_{0}$ are both in $\mathbf{L}_{2}(0, \infty ; Y)$ by assumption. So (8) and (10) hold, yielding

$$
\begin{aligned}
C^{\text {new }} x_{0} & =\left(\bar{B}^{*} \bar{\Psi}^{*} S_{t}^{*} \Psi x_{0}+\bar{D}^{*} \Psi x_{0}\right)(0) \text { using }(9) \\
& =\left(\bar{B}^{*} \bar{\Psi}^{*} \Psi T(t) x_{0}+\bar{D}^{*} C T(t) x_{0}\right)(0) \\
& =\left(\bar{B}^{*} \bar{\Psi}^{*} \Psi+\bar{D}^{*} C\right) x_{0} \quad \operatorname{1sing}(4) .
\end{aligned}
$$

Since all the operators on the right-hand side are bounded, $C^{\text {new }}$ is in $\mathcal{L}(Z, Y)$. The given expression for $V=\bar{\Psi}^{*} \Psi$ follows from (8).

We now introduce some stability notions for bounded state linear systems $\Sigma(A, B, C, D)$, which we also denote $\Sigma(A, B, C)$. 
Definition 2.6 $A C_{0}$-semigroup $T(t)$ on a Hilbert space $Z$ is called strongly stable if

$$
\lim _{t \rightarrow \infty} T(t) x=0 .
$$

Definition 2.7 The bounded linear system $\Sigma(A, B, C)$ is strongly stable if

1. A generates a strongly stable $C_{0}-$ semigroup $T(t)$;

2. $\Psi \in \mathcal{L}\left(Z, \mathbf{L}_{2}(0, \infty ; Y)\right)$;

3. $\tilde{\Phi} \in \mathcal{L}\left(\mathrm{L}_{2}(0, \infty ; U), Z\right)$;

4. $\mathbb{F} \in \mathcal{L}\left(\mathbf{L}_{2}(0, \infty ; U), \mathbf{L}_{2}(0, \infty ; Y)\right)$.

It is well-known (see for instance Weiss [23]) that (ii)-(iv) respectively are equivalent to

(ii) $\ell C(s I-A)^{-1} x \in \mathbf{H}_{2}(Y)$ for all $x \in Z$;

(iii) $B^{*}\left(s I-A^{*}\right)^{-1} z \in \mathbf{H}_{2}(U)$ for all $z \in Z$;

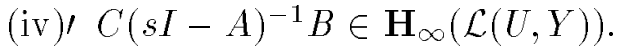

Definition 2.8 The bounded linear system $\Sigma(A, B,-)$ is strongly stabilizable if there exists $F \in \mathcal{L}(Z, U)$ such that $\Sigma\left(A_{F}, B, F\right)$ is a strongly stable bounded linear system, where $A_{F}=A+B F$.

Definition 2.9 The bounded linear system $\Sigma(A, B, C)$ is statically stabilizable if there exists $K \in \mathcal{L}(Y, U)$ such that $\Sigma\left(A_{K C}, B, C\right)$ is a strongly stable bounded linear system, where $A_{K C}=A+B K C$.

Definition 2.10 The operator $X \in \mathcal{L}(Z)$ is a strongly stabilizing solution to the Riccati equation (1), if

1. $X$ satisfies (1) for all $z \in D(A)$;

2. $\Sigma\left(A_{F_{X}}, B,\left(\begin{array}{c}F_{X} \\ C\end{array}\right)\right)$ is a strongly stable bounded linear system;

where $A_{F_{X}}=A+B F_{X}$ and $F_{X}=-R^{-1}\left(B^{*} X+N C\right)$.

We remark that the concept of strong stability (stabilizability) in Definition $2.7(2.8)$ played an essential role in Staffans [19, 20]. The Definitions 2.9 and 2.10 are inspired by these papers, although the concept of static stabilizability introduced here is new. Note that it implies that the system is both stabilizable and detectable in the sense defined in Staffans [19, 20]. 
An essential difference between the development of the Popov approach in Weiss [25] and in Weiss and Weiss [26] lies in the initial formulation of the problem. In Weiss [25], he took $C=I$ (and $N=L$ ) and this forced him to work with exponential stability, instead of strong stability. We show how the extra $C$-factor allows one to weaken the stability requirements. Let us consider the cost functional associated with (1).

$$
J\left(x_{0}, u(\cdot)\right)=\int_{0}^{\infty}\left\langle\left[\begin{array}{cc}
C^{*} Q C & C^{*} N^{*} \\
N C & R
\end{array}\right]\left(\begin{array}{l}
x(t) \\
u(t)
\end{array}\right),\left(\begin{array}{l}
x(t) \\
u(t)
\end{array}\right)\right\rangle_{Z \times U} d t
$$

subject to the constraints

$$
\begin{aligned}
\dot{x} & =A x+B u, \quad x(0)=x_{0} \\
y & =C x .
\end{aligned}
$$

Noting that

$$
y\left(x_{0}, u(\cdot)\right)=\Psi x_{0}+\mathbb{F} u
$$

as in Weiss [25] and Weiss and Weiss [26] we reformulate the cost as

$$
J\left(x_{0}, u\right)=\left\langle\left[\begin{array}{cc}
\Psi^{*} Q \Psi & \Psi^{*}\left(Q \mathbb{F}+N^{*}\right) \\
\left(\mathbb{F}^{*} Q+N\right) \Psi & \mathcal{R}
\end{array}\right]\left(\begin{array}{c}
x_{0} \\
u
\end{array}\right),\left(\begin{array}{c}
x_{0} \\
u
\end{array}\right)\right\rangle_{Z \times L_{2}(0, \infty ; U)},(14
$$

where

$$
\mathcal{R}=R+N \mathbb{F}+\mathbb{F}^{*} N^{*}+\mathbb{F}^{*} Q \mathbb{F} .
$$

If we assume that $\Sigma(A, B, C)$ is a strongly stable bounded linear system, then $\Psi \in \mathcal{L}\left(Z, \mathbf{L}_{2}(0, \infty ; Y)\right)$ and $\mathbb{F} \in \mathcal{L}\left(\mathbf{L}_{2}(0, \infty ; U), \mathbf{L}_{2}(0, \infty ; Y)\right)$ and this is sufficient to ensure that $J$ is well defined for all inputs $u \in \mathrm{L}_{2}(0, \infty ; U)$. If $C=I$, then to ensure that $\Psi$ and $\mathbb{F}$ are bounded, one needs to assume that $T(t)$ is exponentially stable, as was done in Weiss [25]. The introduction of the extra $C$-factor, weakens this assumption to (ii) and (iv) in the definition of strong stability.

We have the following result for strongly stable semigroups.

Lemma 2.11 Let $T(t)$ be a strongly stable $C_{0}$-semigroup and let $B$ be an infinitetime admissible input operator for $T(t)$, i.e. $\tilde{\Phi} \in \mathcal{L}\left(\mathbf{L}_{2}(0, \infty ; U), z\right)$, where $\tilde{\Phi}$ is defined as in (6). Under these assumptions,

$$
\lim _{t \rightarrow \infty} \int_{0}^{t} T(t-s) B u(s) d s=0 .
$$

Proof. We have

$$
\int_{0}^{t} T(t-s) B u(s) d s=T\left(t-t_{1}\right) \int_{0}^{t_{1}} T\left(t_{1}-s\right) B u(s) d s+\int_{t_{1}}^{t} T(t-s) B u(s) d s .
$$


Now, since $T(t)$ is strongly stable and $u(t) \in \mathbf{L}_{2}(0, \infty ; U)$, we have for fixed $t_{1}$,

$$
T\left(t-t_{1}\right) \int_{0}^{t_{1}} T\left(t_{1}-s\right) B u(s) d s \rightarrow 0 \text { as } t \rightarrow \infty .
$$

For the remaining term, we have

$$
\begin{aligned}
\int_{t_{1}}^{t} T(t-s) B u_{*}(s) d s & =\int_{0}^{t} T(t-s) B u_{t_{1}}(s) d s \\
& =\left(\Phi_{t} u_{t_{1}}\right)(t)
\end{aligned}
$$

where we defined

$$
u_{t_{1}}(t)=\left\{\begin{array}{ll}
0 & t \leq t_{1} \\
u_{*}(t) & t>t_{1}
\end{array},\right.
$$

and $\Phi_{t}$ and $\tilde{\Phi}_{t}$ are defined as in (4), (5). It is easy to see that $\left\|\Phi_{t}\right\|=\left\|\tilde{\Phi}_{t}\right\| \leq\|\tilde{\Phi}\|$. Hence,

$$
\left\|\left(\Phi_{t} u_{t_{1}}\right)(t)\right\|_{Z} \leq\|\tilde{\Phi}\|\left(\int_{0}^{\infty}\left\|u_{t_{1}}(s)\right\|^{2} d t\right)^{\frac{1}{2}} .
$$

By assumption, $\|\tilde{\Phi}\|<\infty$, so the above expression can be made arbitrarily small by choosing $t_{1}$ large enough. This proves the claim.

The following concepts play a central role in our theory.

Definition 2.12 The Popov function $\Pi: j \mathbb{R} \rightarrow \mathcal{L}(U)$ associated with the Riccati equation (1) is defined by

$$
\Pi(j \omega)=R+N G(j \omega)+G(j \omega)^{*} N^{*}+G(j \omega)^{*} Q G(j \omega)
$$

where $G(j \omega)=C(j \omega I-A)^{-1} B$ and $\omega \in \mathbb{R}$.

We remark that a sufficient condition for the Popov function $\Pi$ to be well-defined is that $\mathbb{F}$ is a bounded operator from $\mathbf{L}_{2}(0, \infty ; U)$ to $\left.\mathrm{L}_{2}(0, \infty ; Y)\right)$ for $\Sigma(A, B, C)$. In this case, $G \in \mathbf{H}_{\infty}(\mathcal{L}(U, Y))$ and it has an extension to $s=j \omega$ in the sense that $\lim _{\sigma \rightarrow 0} G(\sigma+j \omega) u$ exists for all $u \in U$ and for almost all $\omega \in \mathbb{R}$ (see Theorem 4.5 in Rosenblum and Rovnyak [15]). Moreover, $\Pi \in \mathbf{L}_{\infty}(\mathcal{L}(U))$.

Definition 2.13 A function $\Pi \in \mathbf{L}_{\infty}(\mathcal{L}(U))$ has a Wiener-Hopf factorization if

$$
\Pi(j \omega)=M^{\sim}(j \omega) N(j \omega) \text {, for almost all } \omega \in \mathbb{R},
$$

where $M, N, M^{-1}, N^{-1} \in \mathbf{H}_{\infty}(\mathcal{L}(U))$, and $M^{\sim}(s)=M(-\bar{s})^{*}$. 
A sufficient condition for II to have a Wiener-Hopf factorization is that it satisfies the coercivity condition

$$
\Pi(j \omega) \geq \varepsilon I, \text { for some } \varepsilon>0 \text {, almost all } \omega \in \mathbb{R} \text {. }
$$

See Theorem 5 in Devinatz and Shinbrot [9] or Rosenblum and Rovnyak [15], Theorem 3.7 .

Definition 2.14 Let $\Pi \in \mathbf{L}_{\infty}(\mathcal{L}(Z))$. The Toeplitz operator with symbol $\Pi$ is defined by

$$
\mathcal{T}_{\Pi}: \mathbf{H}_{2}(Z) \rightarrow \mathbf{H}_{2}(Z), \quad \mathcal{T}_{\Pi} x=P_{+} \Pi x, \text { for } x \in \mathbf{H}_{2}(Z),
$$

where $P_{+}$is the orthogonal projection from $\mathbf{L}_{2}(-j \infty, j \infty ; Z)$ onto $\mathbf{H}_{2}(Z)$. The timedomain Toeplitz operator with symbol $\Pi$ is defined by

$$
\mathcal{T}_{\Pi}^{t}: \mathbf{L}_{2}(0, \infty ; Z) \rightarrow \mathbf{L}_{2}(0, \infty ; Z), \quad \mathcal{T}_{\Pi}^{t} x(\cdot)=\mathfrak{F}^{-1} \mathcal{T}_{\Pi}(\mathfrak{F} x(\cdot)),
$$

for $x \in \mathbf{L}_{2}(0, \infty ; Z)$, where $\mathfrak{F}$ denotes the Fourier transformation that maps $\mathbf{L}_{2}(0, \infty ; Z)$ onto $\mathbf{H}_{2}(Z)$.

Theorem A.6.26 in Curtain and Zwart [6] states that $\mathcal{T}_{\Pi}$ and $\mathcal{T}_{\Pi}^{t}$ are bounded operators.

We state some properties of Toeplitz operators, that we will use throughout this paper; proofs can be found in Section 2.1 of Weiss [25].

Lemma 2.15 1. If $G^{\sim} \in \mathbf{H}_{\infty}(\mathcal{L}(Z))$ or $K \in \mathbf{H}_{\infty}(\mathcal{L}(Z))$, then $\mathcal{T}_{G K}=\mathcal{T}_{G} \mathcal{T}_{K}$;

2. If $G \in \mathbf{H}_{\infty}(\mathcal{L}(Z))$, then $\mathcal{T}_{G}$ equals the multiplication map induced by $G$. Consequently, $\mathcal{T}_{G}^{t}=\mathbb{F}_{G}$, where $\mathbb{F}_{G}$ is the extended input-output map of the system represented by $G$;

3. The adjoint of the Toeplitz operator $\mathcal{T}_{G}$ is given by $\mathcal{T}_{G}^{*}=\mathcal{T}_{G^{\sim}}$.

The invertibility of the Toeplitz operator with symbol the Popov function is important in the proof of our main result.

Lemma 2.16 Consider the Toeplitz operator $\mathcal{T}_{\Pi}$ with symbol the Popov function $\Pi$.

1. $\mathcal{R}$ defined by (15) equals the time-domain Toeplitz operator $\mathcal{T}_{\Pi}^{t}$ with symbol the Popov function (17);

2. $\mathcal{R}$ is boundedly invertible if and only $\mathcal{T}_{\Pi}$ is;

3. $\mathcal{T}_{\Pi}$ is boundedly invertible if $\Pi$ admits a Wiener-Hopf factorization; 
Proof. (i) This is proven in Lemma 4.27 in Weiss [25].

(ii) This is a direct consequence of part (i) and the fact that $\mathcal{T}_{\Pi}$ and $\mathcal{T}_{\Pi}^{t}$ are isomorphic.

(iii) See Theorem 5 of Devinatz and Shinbrot [9]

Finally, we conclude this section with a technical lemma, that is proven by Staffans in Theorem 2.7 of [18], Theorem 2.6 of [19], Theorem 13 of [21], as well as in Theorem 11.3 in Weiss and Weiss [26] for the general case of a well-posed linear system. For completeness, we give a proof for the special case of a bounded linear system.

Lemma 2.17 Let $\Sigma(A, B, C)$ be a strongly stable bounded linear system and let the operators $N, Q$ and $R$ be given. Suppose that the corresponding Popov function $\Pi \in \mathbf{L}_{\infty}(\mathcal{L}(U))$ has a Wiener-Hopf factorization, $\Pi(j \omega)=M^{\sim}(j \omega) N(j \omega)$. Then $N(\cdot)$ is the transfer function of the well-posed linear system $\Sigma=\left(\mathbf{T}, \boldsymbol{\Phi}, \boldsymbol{\Psi}_{N}, \mathbf{F}_{N}\right)$, where the $C_{0}$-semigroup and the input map are the same as those for $\Sigma(A, B, C)$.

Proof. Let $\Pi$ have a Wiener-Hopf factorization $\Pi=M^{\sim} N$. This implies that $M$ is invertible over $\mathbf{H}_{\infty}(\mathcal{L}(U))$. We denote the extended input-output map corresponding to the transfer function $M$ by $\mathbb{F}_{M}$. Because $\mathbb{F}_{M}$ and $M$ are isomorphic via the Fourier transform [6, Theorem A.6.27], $\mathbb{F}_{M}^{-1}$ is bounded and shift invariant.

Next, we define

$$
\overline{\mathbb{F}}=\left(Q \mathbb{F}+N^{*}\right) \mathbb{F}_{M}^{-1}
$$

which is a bounded operator from $\mathbf{L}_{2}(0, \infty ; U)$ to $\mathbf{L}_{2}(0, \infty ; Y)$. Because $\mathbb{F}$ and $\mathbb{F}_{M}^{-1}$ are shift-invariant and bounded, and $Q$ and $N$ are bounded, it follows that $\overline{\mathbb{F}}$ is shift-invariant and bounded. So, from Lemma 2.5 , we can define a new output map $\Psi_{N}$ for $T(t)$ by

$$
\left(\Psi_{N} x_{0}\right)(t)=\left(\overline{\mathbb{F}}^{\star} \Psi x_{0}\right)(t)=\left(\left(\mathbb{F}_{M}\right)^{-*}\left(\mathbb{F}^{\star} Q+N\right) \Psi x_{0}\right)(t) .
$$

Next, we have to show that $\mathbb{F}_{N}$ satisfies the functional equation in part (iv) of Definition 2.4:

$$
\left(\mathbb{F}_{N}(u \underset{\tau}{\diamond} v)\right)(t)=\left(\left(\mathbb{F}_{N} u\right) \underset{\tau}{\diamond}\left(\Psi_{N} \Phi_{\tau} u+\mathbb{F}_{N} v\right)\right)(t)
$$

We can split the equation above in two equations, one for $0 \leq t<\tau$ and one for $t>\tau$. The first one is a trivial equation, the second one becomes

$$
\left(\mathbb{F}_{N}(u \underset{\tau}{\diamond} v)\right)(t)=\left(\Psi_{N} \Phi_{\tau} u+\mathbb{F}_{N} v\right)(t-\tau) .
$$

If we apply a shift over a distance $\tau$ to this equation, we see that we must check whether $\mathbb{F}_{N}$ satisfies

$$
S_{\tau}^{*} \mathbb{F}_{N}(u \diamond v)=\Psi_{N} \Phi_{\tau} u+\mathbb{F}_{N} v
$$


where $S_{\tau}$ is the right shift on $\mathrm{L}_{2}(0, \infty ; U)$. Now, since $\Sigma=(\mathbf{T}, \mathbf{\Phi}, \Psi, \mathbf{F})$ is a wellposed linear system,

$$
S_{\tau}^{*} \mathbb{F}(u \diamond v)=\Psi \Phi_{\tau} u+\mathbb{F} v,
$$

and so

$$
\overline{\mathbb{F}}^{*} S_{\tau}^{*} \mathbb{F}(u \diamond v)=\overline{\mathbb{F}}_{\tau}^{*} \Psi \Phi_{\tau} u+\overline{\mathbb{F}^{*}} \mathbb{F} v .
$$

$\therefore$ From the shift-invariance of $\overline{\mathbb{F}}$, we obtain

$$
S_{\tau}^{*} \overline{\mathbb{A}}^{*} \mathbb{F}(u \diamond v)=\overline{\mathbb{F}^{*}} \Psi \Phi_{\tau} u+\overline{\mathbb{F}^{*}} \mathbb{F} v .
$$

Next,

$$
\begin{aligned}
\mathcal{T}_{\Pi}^{t} & =\mathcal{T}_{M}^{t} \sim N \\
& =\mathcal{R} \text { by Lemmas } 2.15 \text { and } 2.16(\mathrm{i}) \\
& =\left(\mathbb{F}^{*} Q+N\right) \mathbb{F}+\mathbb{F}^{*} N^{*}+R \text { from }(15) \\
& =\mathbb{F}_{M}^{*} \overline{\mathbb{F}^{*}} \mathbb{F}+\mathbb{F}^{*} N^{*}+R \text { from }(20) .
\end{aligned}
$$

By Lemma $2.15, \mathcal{T}_{\Pi}^{t}=\mathcal{T}_{M}^{t} \sim \mathcal{T}_{N}^{t}=\mathbb{F}_{M}^{*} \mathbb{F}_{N}^{*}$, which in combination with the above shows that

$$
\mathbb{F}_{M}^{*}\left(\mathbb{F}_{N}-\overline{\mathbb{H}}^{*} \mathbb{H}^{*}\right)=\mathbb{H}^{*} N^{*}+R,
$$

and since $\mathbb{F}_{M}$ is invertible we obtain

$$
\mathbb{F}_{N}-\overline{\mathbb{H}}^{*} \mathbb{F}=\mathbb{F}_{M}^{-*}\left(\mathbb{H}^{*} N^{*}+R\right) .
$$

Using a similar argument as that for $\overline{\mathbb{F}}$ you can show that the right-hand side is invariant with respect to the left shift $S_{\tau}^{*}$, and so the same holds for the left-hand side. Therefore,

$$
\begin{aligned}
S_{\tau}^{*}\left(\mathbb{F}_{N}-\overline{\mathbb{F}}^{*} \mathbb{F}\right)(u \underset{\tau}{\diamond} v) & =\left(\mathbb{F}_{N}-\overline{\mathbb{F}}^{*} \mathbb{F}\right) S_{\tau}^{*}(u \diamond v) \\
& =\left(\mathbb{F}_{N}-\overline{\mathbb{F}}^{*} \mathbb{F}\right) v,
\end{aligned}
$$

and so

$$
\begin{aligned}
& S_{\tau}^{*} \mathbb{F}_{N}(u \diamond v)=S_{\tau}^{*} \overline{\mathbb{F}^{*}} \mathbb{F}(u \diamond v)+\left(\mathbb{F}_{N}-\overline{\mathbb{F}^{*}} \mathbb{F}^{\top}\right) v \\
& =\overline{\mathbb{H}}^{*} \Psi \Phi_{\tau} u+\overline{\mathbb{F}}^{*} \mathbb{F} v+\mathbb{F}_{N} v-\overline{\mathbb{F}^{*}} \mathbb{F}^{*} v \text { using }(22) \\
& =\overline{\mathbb{H}}^{*} \Psi \Phi_{\tau} u+\mathbb{F}_{N} v \\
& =\Psi_{N} \Phi_{\tau} u+\mathbb{F}_{N} v \text { from (22). }
\end{aligned}
$$

This shows that $\left(\mathbf{T}, \boldsymbol{\Phi}, \Psi_{N}, \mathbf{F}_{N}\right)$ is a well-posed linear system with the transfer function $N(s)$.

In the general theory in Weiss and Weiss [26], neither $N$ nor $M$ need to be regular. however, in the case of a bounded linear system, $N$ is always regular, since it has the same bounded input operator $B$. 


\section{Riccati equation for strongly stable systems.}

Now, we can prove our main result which relates strongly stabilizing solutions of the Riccati equation to Wiener-Hopf factorizations. The sufficiency part is contained in Theorem 30 of Staffans [21].

Theorem 3.1 Consider the strongly stable bounded linear system $\Sigma(A, B, C)$ and the operators $N, Q, R$, satisfying the assumptions A1-A4. There exists a unique, self-adjoint strongly stabilizing solution to the Riccati equation (1) if and only if the associated Popov function $\Pi$ has a Wiener-Hopf factorization.

Proof. Necessity: Suppose that the Riccati equation has a strongly stabilizing solution $X$ with corresponding feedback operator $F_{X}$. Then, with a long but straightforward calculation, using the fact that $X$ satisfies the Riccati equation, we can check that the following expression holds:

$$
\Pi(j \omega)=\left(I-F_{X}(j \omega I-A)^{-1} B\right)^{*} R\left(I-F_{X}(j \omega I-A)^{-1} B\right) .
$$

We define $N(s)=I-F_{X}(s I-A)^{-1} B, M(s)=R N(s)$. To show that $N$ and $M$ form a Wiener-Hopf factorization of $\Pi$, it is sufficient to show that $N(s) \in \mathbf{H}_{\infty}(\mathcal{L}(U))$ and $N^{-1}(s) \in \mathbf{H}_{\infty}(\mathcal{L}(U))$, because the invertibility of $R$ shows that then the same holds for $M$ and $M^{-1}$.

¿From the definition of a strongly stabilizing solution, $F_{X}\left(s I-A_{F_{X}}\right)^{-1} B \in$ $\mathbf{H}_{\infty}(\mathcal{L}(U))$ and so $N^{-1}(s)=I+F_{X}\left(s I-A_{F_{X}}\right)^{-1} B \in \mathbf{H}_{\infty}(\mathcal{L}(U))$. To prove that $N \in \mathbf{H}_{\infty}(\mathcal{L}(U))$, note that $B^{*}\left(s I-A^{*}\right)^{-1} z \in \mathbf{H}_{2}(U)$ implies that $\left(I-N^{\sim}(-s)\right) u=$ $B^{*}\left(s I-A^{*}\right)^{-1} F_{X}^{*} u$ is in $\mathbf{H}_{2}(U)$ for all $u \in U$. Moreover, $N(s)=\left(M^{\sim}\right)^{-1} \Pi \in$ $\mathbf{L}_{\infty}(\mathcal{L}(U))$ and consequently $N^{\sim}(-s) \in \mathbf{L}_{\infty}(\mathcal{L}(U))$, as well. So, by Corollary 2.3, $N(s) \in \mathbf{H}_{\infty}(\mathcal{L}(U))$.

Sufficiency: By assumption $\Sigma(A, B, C)$ is strongly stable. Suppose that $\Pi$ has a Wiener-Hopf factorization, $\Pi(j \omega)=M^{\sim}(j \omega) N(j \omega)$. By Lemma 2.16(iii) $\mathcal{T}_{\Pi}$ is boundedly invertible and by part (ii) of the same lemma it follows that $\mathcal{R}$ is boundedly invertible. As in the proof of Theorem 2.15 in Weiss [25], you can show that the self-adjoint operator $X$ defined by

$$
X=\Psi^{*} Q \Psi-\Psi^{*}\left(Q \mathbb{F}+N^{*}\right) \mathcal{R}^{-1}\left(\mathbb{F}^{*} Q+N\right) \Psi
$$

is in $\mathcal{L}(Z)$ and satisfies the Riccati equation (1) and moreover, $u_{*} \in \mathrm{L}_{2}(0, \infty ; U)$ and $y_{*} \in \mathbf{L}_{2}(0, \infty ; Y)$, where

$$
\begin{aligned}
u_{*}(\cdot) & =-R^{-1}\left(B^{*} X+N C\right) x_{*}(\cdot)=F_{X} T_{F_{X}}(\cdot) x_{0} \\
& =-\mathcal{R}^{-1}\left(\mathbb{F}^{*} Q+N\right) \Psi x_{0} \\
y_{*}(\cdot) & =C x_{*}(\cdot)=C T_{F_{X}}(\cdot) x_{0}=\Psi x_{0}+\mathbb{F} u_{*}(\cdot)
\end{aligned}
$$

Because of the $C$-factor we cannot conclude exponential stability ifrom (25), as was done in Weiss $[24,25]$, but we can show that $T_{F_{X}}$ is a strongly stable semigroup, by 
considering the perturbations formula from Curtain and Zwart [6, Theorem 3.2.1]:

$$
\begin{aligned}
T_{F_{X}}(t) x_{0} & =T(t) x_{0}-\int_{0}^{t} T(t-s) B R^{-1}\left(B^{*} X+N C\right) T_{F_{X}}(s) x_{0} d s \\
& =T(t) x_{0}+\int_{0}^{t} T(t-s) B u_{*}(s) d s \text { using }(24)
\end{aligned}
$$

Now, since $T(t)$ is strongly stable and $u_{*}(t) \in \mathrm{L}_{2}(0, \infty ; U)$, Lemma 2.11 shows that the right-hand side tends to zero as $t$ goes to infinity. Hence, $T_{F_{X}}(t)$ is a strongly stable semigroup.

It remains to show the property (2) in the definition of a strongly stabilizing solution. It follows directly from the equations (24) and (25) that $F_{X}\left(s I-A_{F_{X}}\right)^{-1} x_{0} \in$ $\mathbf{H}_{2}(U)$ and $C\left(s I-A_{F_{X}}\right)^{-1} x_{0} \in \mathbf{H}_{2}(Y)$. Using the perturbation formulas

$$
\begin{aligned}
& B^{*}\left(s I-A_{F_{X}}^{*}\right)^{-1} z=B^{*}\left(s I-A^{*}\right)^{-1} z+B^{*}\left(s I-A_{F_{X}}^{*}\right)^{-1} F_{X}^{*} \cdot B^{*}\left(s I-A^{*}\right)^{-1} z \\
& C\left(s I-A_{F_{X}}\right)^{-1} B=C(s I-A)^{-1} B+C(s I-A)^{-1} B \cdot F_{X}\left(s I-A_{F_{X}}\right)^{-1} B
\end{aligned}
$$

and the facts that $B^{*}\left(s I-A^{*}\right)^{-1} z \in \mathbf{H}_{2}(U)$, for all $z$ in $Z$ and that $C(s I-$ $A)^{-1} B \in \mathbf{H}_{\infty}(\mathcal{L}(U, Y))$, it follows that $B^{*}\left(s I-A_{F_{X}}^{*}\right)^{-1} z \in \mathbf{H}_{2}(U)$ and $C(s I-$ $\left.A_{F_{X}}\right)^{-1} B \in \mathbf{H}_{\infty}(\mathcal{L}(U, Y))$ if $B^{*}\left(s I-A_{F_{X}}\right)^{-1} F_{X}^{*} \in \mathbf{H}_{\infty}(\mathcal{L}(U))$ or, equivalently, $F_{X}\left(s I-A_{F_{X}}\right)^{-1} B \in \mathbf{H}_{\infty}(\mathcal{L}(U))$. Therefore, it suffices to prove this last assertion.

¿From Lemma 2.17 we have that $N$ has a realization $\left(\mathbf{T}, \boldsymbol{\Phi}, \Psi_{N}, \mathbf{F}_{N}\right)$ as a wellposed linear system. It follows :from this that it has $A$ as generator of its semigroup and that $B$ is its input operator. Because $B$ is bounded, $N$ actually has a realization as a regular linear system, with generating operators $\left(A, B, C_{N}, D_{N}\right)$, see Weiss [23, Theorem 5.8]. If we normalize $N$ such that $\lim _{\lambda \rightarrow \infty} N(\lambda) u=u$ (this can be done because $N$ is invertible), then clearly $D_{N}=I$. To find $C_{N}$, note that $\mathcal{R}^{-1}\left(\mathbb{F}^{*} Q+\right.$ N) $\Psi x_{0}=R^{-1}\left(B^{*} X+N C\right) T_{F_{X}}(\cdot) x_{0}$ and $\mathcal{R}=\mathbb{F}_{M}^{*} \mathbb{F}_{N}$. Thus, using the defining equation (21) for $\Psi_{N}, \mathbb{F}_{N}^{-1} \Psi_{N} x_{0}=R^{-1}\left(B^{*} X+N C\right) T_{F_{X}}(\cdot) x_{0}$ and

$$
\left(\Psi_{N} x_{0}\right)(t)=\mathbb{F}_{N}\left(R^{-1}\left(B^{*} X+N C\right) T_{F_{X}}(\cdot) x_{0}\right)(t) .
$$

Using the regularity of $\mathbb{F}_{N}$, with $D=I$, and taking $t=0$ in the above equation we see immediately that

$$
C_{N} x_{0}=R^{-1}\left(B^{*} X+N C\right) x_{0}=-F_{X} x_{0} .
$$

So $N(s)=I-F_{X}(s I-A)^{-1} B$. Because $N$ is a Wiener-Hopf factor, it follows that $N^{-1}(s)=I+F_{X}\left(s I-A_{F_{X}}\right)^{-1} B \in \mathbf{H}_{\infty}(\mathcal{L}(U))$. Hence, we can conclude that $X$ is a strongly stabilizing solution. The uniqueness follows as in Lemma 2.13 of Weiss [25].

In the coercive case, we obtain the following corollary. 
Corollary 3.2 Consider the strongly stable bounded linear system $\Sigma(A, B, C)$ and the operators $N, Q, R$, satisfying the assumptions A1-A4. Assume that $R \geq \mu I$ for some $\mu>0$ (i.e. $R$ is coercive). There exists a unique self-adjoint strongly stabilizing solution to the Riccati equation (1) if and only if the associated Popov function $\Pi$ is coercive.

Proof. Sufficiency: Assume that $\Pi$ is coercive. Theorem 3.7 in Rosenblum and Rovnyak [15], shows that $\Pi$ has a Wiener-Hopf factorization. Theorem 3.1 then implies that (1) has a unique self-adjoint strongly stabilizing solution.

Necessity: Suppose (1) has a self-adjoint strongly stabilizing solution $X$, with corresponding feedback $F_{X}$. In the proof of Theorem 3.1 it is shown that

$$
\Pi(j \omega)=S^{\sim}(j \omega) R S(j \omega)
$$

where $S(s)=I-F_{X}(s I-A)^{-1} B$ and $S, S^{-1} \in \mathbf{H}_{\infty}(\mathcal{L}(U))$. By assumption $R$ is coercive and because $S$ is invertible over $\mathbf{H}_{\infty}(\mathcal{L}(U))$, $\Pi$ is coercive, as well.

Another important special case of Theorem 3.1 is the application to J-spectral factorizations for systems that are not exponentially stabilizable. In this case, the coercivity assumption (19) is not satisfied.

Define for Hilbert spaces $Z_{+}, Z_{-}$and a real $\gamma>0$,

$$
J_{Z_{+}, Z_{-}}(\gamma)=\left[\begin{array}{cc}
I_{Z_{+}} & 0 \\
0 & -\gamma^{2} I_{Z_{-}}
\end{array}\right]
$$

Definition 3.3 Suppose that $G \in \mathrm{L}_{\infty}(\mathcal{L}(U, Y))$, where $U=U_{+} \times U_{-}$and $Y=$ $Y_{+} \times U_{-}$and $U_{+}, Y_{+}, U_{-}$are separable Hilbert spaces. A J-spectral factorization of $G$ is defined by

$$
G^{\sim}(j \omega) J_{Y_{+}, U_{-}}(\gamma) G(j \omega)=W^{\sim}(j \omega) J_{U_{+}, U_{-}}(\gamma) W(j \omega)
$$

where $W$ and $W^{-1} \in \mathbf{H}_{\infty}(\mathcal{L}(U))$ and $W$ and $W^{-1}$ are regular.

Theorem 3.4 Suppose that $G(s)$ is the transfer function of the strongly stable bounded linear system $\Sigma(A, B, C, D)$, where $A$ is an operator on $Z, \in \mathcal{L}(U, Z), C \in$ $\mathcal{L}(Z, Y)$ and $U=U_{+} \times U_{-}, Y=Y_{+} \times U_{-}$. Then $G$ has a J-spectral factorization if and only if

1. there exists a boundedly invertible operator $W_{\infty} \in \mathcal{L}\left(Y_{+} \times U_{-}\right)$such that

$$
D^{*} J_{Y_{+}, U_{-}}(\gamma) D=W_{\infty}^{*} J_{U_{+}, U_{-}}(\gamma) W_{\infty}
$$

and 
2. The Riccati equation (1) with $Q=J_{Y_{+}, U_{-}}(\gamma), N=D^{*} J_{Y_{+}, U_{-}}(\gamma), R=$ $D^{*} J_{Y_{+}, U_{-}}(\gamma) D$ has a unique self-adjoint, strongly stabilizing solution $X$. In this case, a J-spectral factor for $G$ is given by

$$
W(s)=W_{\infty}+L(s I-A)^{-1} B,
$$

where $L=J_{U_{+}, U_{-}}(\gamma)^{-1} W_{\infty}^{-*}\left(B^{*} X+D^{*} J_{Y_{+}, U_{-}}(\gamma) C\right)$.

Proof. For convenience of notation we write $J_{U}=J_{U_{+}, U_{-}}(\gamma), J_{Y}=J_{Y_{+}, U_{-}}(\gamma)$.

Sufficiency: The formula for $W(s)$ follows by direct substitution. It remains to prove that $W(s), W^{-1}(s) \in \mathbf{H}_{\infty}(\mathcal{L}(U))$. We define $\tilde{W}(s)=W_{\infty}^{-1} W(s)$ and show that $\tilde{W}$ and $\tilde{W}^{-1} \in \mathbf{H}_{\infty}(\mathcal{L}(U))$. For the Riccati feedback $F_{X}$, we have $F_{X}=$ $-\left(W_{\infty}^{*} J_{U} W_{\infty}\right)^{-1}\left(B^{*} X+D^{*} J_{Y} C\right)$ and furthermore, $\tilde{W}=I-F_{X}(s I-A)^{-1} B$ and $\tilde{W}^{-1}(s)=I+F_{X}\left(s I-A_{F_{X}}\right)^{-1} B$. Because $X$ is a strongly stabilizing solution, $\tilde{W}^{-1} \in$ $\mathbf{H}_{\infty}(\mathcal{L}(U))$. Because $\Sigma(A, B, C, D)$ is strongly stable, $G \in \mathbf{H}_{\infty}(\mathcal{L}(U, Y))$. This implies that $G^{\sim} J_{Y} G \in \mathrm{L}_{\infty}(\mathcal{L}(U))$ and consequently that $\tilde{W}^{\sim}=G^{\sim} J_{Y} G W^{-1} J_{U} W_{\infty}^{-1} \in$ $\mathbf{L}_{\infty}(\mathcal{L}(U))$. The strong stability of $\Sigma(A, B, C, D)$ also implies that $\left(I-W^{\sim}(-s)\right) u=$ $B^{*}\left(s I-A^{*}\right)^{-1} F_{X}^{*} u \in \mathbf{H}_{2}(U)$ for all $u \in U$ So, we can conclude by Corollary 2.3 that $W(s) \in \mathbf{H}_{\infty}(\mathcal{L}(U))$.

Necessity: Conversely, suppose that $G$ has a J-spectral factorization.

(i) Let the limit of the spectral factor $W$ along the positive real axis be

$$
\lim _{\lambda \rightarrow \infty} W(\lambda)=W_{\infty}
$$

Note that in the definition of a J-spectral factorization, we demanded that $W$ be regular. Hence, this limit exists. Then, taking limits on both sides in (26), we ob$\operatorname{tain} D^{*} J_{Y} D=W_{\infty}^{*} J_{U} W_{\infty}$. (ii) We define $\Pi=G^{\sim} J_{Y} G$. Note that $\Pi$ is the Popov function associated with the Riccati equation (1) with $Q=J_{Y}, N=D^{*} J_{Y}, R=$ $D^{*} J_{Y} D$. Setting $M(s)=W(s), N(s)=J_{U} W(s)$, we obtain a Wiener-Hopf factorization of $\Pi$. Consequently, the Riccati equation has a unique self-adjoint, strongly stabilizing solution.

\section{Riccati equation for unstable systems.}

The following lemma shows that in certain cases, we can reduce the unstable case to the stable case.

Lemma 4.1 $1 . X=X^{*} \in \mathcal{L}(Z)$ is a solution to the Riccati equation (1) if and only if $X$ is a solution to the feedback Riccati equation (28)

$$
\begin{gathered}
A_{F}^{*} X z+X A_{F} z-\left(B^{*} X+N C+R F\right)^{*} R^{-1}\left(B^{*} X+N C+R F\right) z \\
\quad+\left(C^{*} Q C+C^{*} N^{*} F+F^{*} N C+F^{*} R F\right) z=0
\end{gathered}
$$

for all $z \in D(A)$, where $A_{F}=A+B F$. 
2. $X$ is a strongly stabilizing solution for (1) if and only if it is a strongly stabilizing solution for (28). Furthermore, the generator of the strongly stable semigroup is the same in both cases.

3. There exists at most one strongly stabilizing solution.

4. The Popov function $\Pi$ associated with (1) is related to the Popov function $\Pi_{F}$ associated with (28) by

$$
\begin{aligned}
\Pi_{F}(j \omega) & =R+(N C+R F)\left(j \omega I-A_{F}\right)^{-1} B \\
& +B^{*}\left(-j \omega I-A_{F}^{*}\right)^{-1}\left(F^{*} R+C^{*} N^{*}\right) \\
& +B^{*}\left(-j \omega I-A_{F}^{*}\right)^{-1}\left(F^{*} N C+C^{*} N^{*} F+F^{*} R F+C^{*} Q C\right) . \\
& \left(j \omega I-A_{F}\right)^{-1} B \\
= & S_{F}(j \omega) \Pi(j \omega) S_{F}(j \omega)
\end{aligned}
$$

for $j \omega \in \rho(A) \cap \rho\left(A_{F}\right)$, where $S_{F}(s)=I+F\left(s I-A_{F}\right)^{-1} B$.

5. $\Pi(j \omega) \geq \varepsilon I_{U}$ if and only if $\Pi_{F}(j \omega) \geq \varepsilon I_{U}$.

Proof. (i), (ii), (iv), (v) were established in Weiss [24, Lemmas 7 and 8],[25]. (iii) is proved as in Lemma 2.13 in Weiss [25]; the exponential stability was not necessary. (see also Curtain and Zwart [8]). The main point is that $A_{F_{X}}=A-B R^{-1}\left(B^{*} X+\right.$ $N C)=A_{F}-B R^{-1}\left(B^{*} X+N C+R F\right)$.

To retain the original form of the Riccati equation, we must use feedbacks of the form $F=K C$. Hence, we can reduce the unstable case to the stable case if $\Sigma(A, B, C)$ is statically stabilizable. The sufficiency part of the following theorem is included in Theorem 30 of Staffans [21].

Theorem 4.2 Consider the Riccati equation (1) under the assumptions A1-A4, and assume that the bounded linear system $\Sigma(A, B, C)$ is statically stabilizable by a static output feedback $u=K y$.

1. (1) has a unique self-adjoint, strongly stabilizing solution if and only if $\Pi_{K C}$ has a Wiener-Hopf factorization.

2. Let $R \geq \mu I$ for some $\mu>0$. (1) possesses a unique self-adjoint, strongly stabilizing solution $X \in \mathcal{L}(X)$ if and only if $\Pi$ is coercive (i.e. $\Pi$ satisfies (19)).

Proof. (i) Theorem 3.1 shows us that the feedback Riccati equation (28) has a unique self-adjoint, strongly stabilizing solution if and only if $\Pi_{K C}$ has a WienerHopf factorization. Lemma 4.1(i-ii), shows that the feedback Riccati equation (28) has a unique self-adjoint, strongly stabilizing solution if and only if the Riccati equation (1) has. 
(ii) Sufficiency: Assume that $\Pi$ is coercive. Since the system is statically stabilizable, we can introduce the feedback system with $F=K C$ to obtain the stable system $\Sigma\left(A_{K C}, B, C\right)$ and its corresponding Riccati equation (28) with $F=K C$. Lemma 4.1 shows that (1) has a solution if and only if (28) does and from Theorem $3.1,(28)$ has a unique strongly stabilizing solution if $\Pi_{K C}$ given by (29) with $F=K C$ is coercive. Now from $(30)$ we have

$$
\Pi_{K C}(j \omega)=S_{K C}^{\sim}(j \omega) \Pi(j \omega) S_{K C}(j \omega) \text {. }
$$

where $S_{K C}(j \omega)=I+K C\left(j \omega I-A_{K C}\right)^{-1} B$. Because $S_{K C}$ and $S_{K C}^{-1}$ are both bounded, $\Pi_{K C}$ is coercive if and only $\Pi$ is. Corollary 3.2 now shows that (28) has a unique, self-adjoint, strongly stabilizing solution $X \in \mathcal{L}(Z)$. By Lemma 4.1, equation (1) has the same solution.

Necessity: Suppose that (1) has a self-adjoint, strongly stabilizing solution. A simple calculation shows that if $F_{X}=-R^{-1}\left(B^{*} X+N C\right)$, its Popov function

$$
\Pi_{F_{X}}(j \omega)=R \geq \mu I,
$$

where the inequality holds by assumption. Thus $\Pi_{F_{X}}$ and consequently $\Pi$ are coercive.

\section{$5 \quad$ Examples}

In this section we will illustrate the results of the preceding sections by applying them to a number of problems. Rather than considering the most general class of systems possible, in this section we specialize to systems of the special structure $\Sigma\left(A, B, B^{*}, D\right)$. As we mentioned in the introduction, our main motivation for writing this paper comes from these systems. They are often used to model flexible structures, such as in Example 1.1. It will turn out that the conditions for existence of solutions of the Riccati equations can in many cases be easily checked for this class of systems.

We will consider Riccati equations for systems $\Sigma\left(A, B, B^{*}, D\right)$ under the assumptions A1-A4 and the additional assumptions

A5. $C=B^{*}$;

A6. $A=A_{0}-B V B^{*}$, where $A_{0}$ generates a contraction semigroup and $V \in \mathcal{L}(U)$;

A7. $A_{0}$ has compact resolvent;

A8. $\Sigma\left(A,-, B^{*}\right)$ is approximately observable. 
We will start by showing that this class of systems is statically stabilizable. In Curtain and Zwart [8] it is shown that A7-A9 imply that

$$
\int_{0}^{\infty}\left\|B^{*} T_{B}(t) x\right\|^{2} d t<\infty, \quad \int_{0}^{\infty}\left\|B^{*} T_{B}^{*}(t) x\right\|^{2} d t<\infty,
$$

for all $x \in Z$, where $T_{B}(t)$ is the $C_{0}$-semigroup generated by $A_{B}:=A_{0}-B B^{*}$. This is equivalent to $B^{*}(s I-A)^{-1} x \in \mathbf{H}_{2}(u), B^{*}\left(s I-A^{*}\right)^{-1} x \in \mathbf{H}_{2}(U)$ for all $x \in Z$. Furthermore, results in Benchimol [3], Arendt and Batty [1] and Balakrishnan [2] show that A6-A8 imply the strong stability of $T_{B}(t)$. A5, A6 imply that $B^{*}(s I-$ $\left.A_{0}\right)^{-1} B$ is positive real. The main result of Curtain and van Keulen [5] shows that in that case $B^{*}\left(s I-A_{B}\right)^{-1} B \in \mathbf{H}_{\infty}(\mathcal{L}(U))$. Concluding, we see that $\Sigma\left(A_{B}, B, B^{*}\right)$ is strongly stable. Hence, $\Sigma\left(A, B, B^{*}\right)$ is statically stabilizable (choose $K=V-I$ in the definition of static stabilizability).

Note that our Example 1.1 satisfies the assumptions A1-A8. We proceed with the application of our main results to the LQ Riccati equation, the strict positive real lemma and the strict bounded real lemma for the class of systems $\Sigma\left(A, B, B^{*}, D\right)$ under the assumptions A1-A8.

\subsection{The standard LQ case}

In addition to A1-A8, we take $R=R^{*} \geq \mu I, Q=Q^{*} \geq 0$ and $N=0$. We consider the Riccati equation for $z \in D(A)$,

$$
A^{*} X z+X A z-X B R^{-1} B^{*} X z+B Q B^{*} z=0,
$$

Under these assumptions, the coercivity assumption (19) is automatically satisfied. Hence, by part 2 of Theorem 4.2 the Riccati equation (32) has a unique self-adjoint, strongly stabilizing solution. This case was also studied in Curtain and Zwart [8] under the additional assumption that $Q$ be coercive. Thus we have reproven the result in Curtain and Zwart [8] for a slightly more general Riccati equation.

\subsection{Strict Positive Real Lemma}

Let $Q=0, N=I, R=D+D^{*}>\varepsilon I>0$ for some $D \in \mathcal{L}(U)$ and assume that $V+V^{*} \geq 0$. Note that in this case the coercivity condition (19) is automatically satisfied. Hence, by part 2 of Theorem 4.2, we have that the Riccati equation

$$
A^{*} X+X A+(X B+B)\left(D+D^{*}\right)^{-1}\left(B^{*} X+B\right)=0
$$

has a unique self-adjoint, strongly stabilizing solution $X$.

The strict positive real lemma corresponds to the case in which $\Sigma\left(A, B, B^{*}\right)$ is strongly stable. In this case, we have the following spectral factorization for $\operatorname{Re}(s)>0$.

$$
G(s)+G^{\sim}(s)=M^{\sim}(s) M(s),
$$


where

$$
\begin{aligned}
G(s) & =D+B^{*}(s I-A)^{-1} B \\
M(s) & =R^{\frac{1}{2}}+R^{-\frac{1}{2}}\left(B^{*}+B^{*} X\right)(s I-A)^{-1} B \\
M^{-1}(s) & =R^{-\frac{1}{2}}-R^{-1}\left(B^{*}+B^{*} X\right)\left(s I-A_{F_{X}}\right)^{-1} B R^{-\frac{1}{2}},
\end{aligned}
$$

where $A_{F_{X}}=A-B R^{-1}\left(B^{*}-B^{*} X\right)$. The spectral factorization (33) has the property that $M(s)$ and $M^{-1}(s) \in \mathbf{H}_{\infty}(\mathcal{L}(U))$.

\subsection{The Strict Bounded Real Lemma}

Let, $Q=-I, N=-D^{*}$ and $R=I-D^{*} D \geq \varepsilon>0$ for some $D \in \mathcal{L}(U)$ and consider the following Riccati equation for $z \in D(A)$

$$
A^{*} X z+X A z-\left(B^{*} X-D^{*} B^{*}\right)^{*} R^{-1}\left(B^{*} X-D^{*} B^{*}\right) z-B B^{*} z=0 .
$$

Theorem 3.1 now implies that (37) possesses a unique strongly stabilizing, selfadjoint solution if and only if

$$
\Pi(j \omega)=I-G(j \omega)^{*} G(j \omega) \geq \mu I
$$

where $G(s)=D+B^{*}(s I-A)^{-1} B$ and $\mu>0$.

The strict bounded real lemma concerns the case for which $\Sigma\left(A, B, B^{*}\right)$ is a strongly stable system. In this case the following spectral factorization is readily verified

$$
I-G^{\sim} G=M^{\sim} M,
$$

with

$$
\begin{aligned}
M(s) & =R^{\frac{1}{2}}+R^{-\frac{1}{2}}\left(B^{*} X-D^{*} B^{*}\right)(s I-A)^{-1} B \\
M^{-1}(s) & =R^{-\frac{1}{2}}-R^{-1}\left(B^{*} X-D^{*} B^{*}\right)\left(s I-A_{F_{X}}\right)^{-1} B R^{-\frac{1}{2}}
\end{aligned}
$$

and $A_{F_{X}}=A-B R^{-1}\left(B^{*} X-D^{*} B^{*}\right)$. Again, $M(s)$ and $M^{-1}(s)$ are in $\mathbf{H}_{\infty}(\mathcal{L}(U))$.

We remark that the above examples can be generalized to systems $\Sigma\left(A, B S, R B^{*}, D\right)$, where $S$ and $R$ are arbitrary invertible operators on $\mathcal{L}(U)$.

Acknowledgments: We are grateful to Olof Staffans for his helpful comments.

\section{References}

[1] W. Arendt and C.J.K. Batty. Tauberian theorems and stability of oneparameter semigroups. Transactions of the American Mathematical Society, 306:837-841, 1988. 
[2] A.V. Balakrishnan. Strong stabilizability and the steady state Riccati equation. Applied Mathematics and Optimization, 7:335-345, 1981.

[3] C.D. Benchimol. A note on weak stabilizability of contraction semigroups. SIAM journal on Control and Optimization, 16:373-379, 1978.

[4] F. Callier and J. Winkin. LQ-optimal control of infinite-dimensional systems by spectral factorization. Automatica, 28:757-770, 1992.

[5] R.F. Curtain and B. van Keulen. Robust control with respect to coprime factors of infinite-dimensional positive real systems. IEEE Transactions on Automatic Control, 37(6):868-871, 1992.

[6] R.F. Curtain and H.J. Zwart. An Introduction to Infinite-Dimensional Linear Systems Theory. Springer-Verlag New York, 1995.

[7] R.F. Curtain and H.J. Zwart. Riccati equations and normalized coprime factorizations for strongly stabilizable systems. Technical Report R-9502, University of Groningen, 1995.

[8] R.F. Curtain and H.J. Zwart. Riccati equations and normalized coprime factorizations for strongly stabilizable infinite-dimensional systems. Systems and Control Letters, 28:11-22, 1996.

[9] A. Devinatz and M. Shinbrot. General Wiener-Hopf operators. Transactions of the American Mathematical Society, 145:467-494, 1969.

[10] P.L. Duren. Theory of $\mathbf{H}^{p}$ Spaces. Academic Press, New York, 1970.

[11] S. Hansen and G. Weiss. New results on the operator Carleson measure criterion. IMA Journal of Mathematical Control and Information, (to appear).

[12] S.M. Joshi. Control of Large Flexible Space Structures, volume 131 of Lecture Notes in Control and Information Sciences. Springer Verlag, Berlin, 1989.

[13] V. Kučera. New results in state estimation and regulation. Automatica, 17:745$748,1981$.

[14] B.P. Molinari. Equivalence relation for the algebraic Riccati equation. SIAM Journal on Control, 11:272-285, 1973.

[15] M. Rosenblum and J. Rovnyak. Hardy Classes and Operator Theory. Oxford University Press, New York, 1985.

[16] M. Slemrod. Feedback stabilization of a linear control system in Hilbert space with an a priori bounded control. Mathematics of Control, Signals and Systems, $2: 265-285,1989$. 
[17] O.J. Staffans. The nonstandard quadratic cost minimization problem for wellposed linear systems. submitted in 1996.

[18] O.J. Staffans. Quadratic optimal control of stable well-posed linear systems. Transactions of the American Mathematical Society, 1996. (to appear).

[19] O.J. Staffans. Coprime factorizations for well-posed linear systems. SIAM Journal of Control and Optimization. (to appear).

[20] O.J. Staffans. Quadratic optimal control of well-posed linear systems. SIAM Journal of Control and Optimization. (to appear).

[21] O.J. Staffans. Critical controls and Riccati equations for abstract linear systems. Technical report, Helsinki University of Technology, Dec. 1995.

[22] O.J. Staffans. On the discrete and continuous time infinite-dimensional Riccati equations. System and Control Letters, 29:131-138, 1996.

[23] G. Weiss. Transfer functions of regular linear systems, part 1: Characterizations of regularity. Transactions of the American Mathematical Society, 342:827-854, 1994.

[24] M. Weiss. Necessary and sufficient conditions for the existence of the stabilizing solution of the Riccati equation in a Hilbert space: a Popov function approach. Journal of Mathematical Systems, Estimation and Control, (to appear).

[25] M. Weiss. Riccati Equations in Hilbert Spaces: A Popov function approach. PhD thesis, Rijksuniversiteit Groningen, The Netherlands, 1994.

[26] M. Weiss and G. Weiss. Optimal control of stable weakly regular linear systems. (manuscript).

[27] Y. You. Dynamical boundary control of two-dimensional Petrovsky system: Vibrating rectangular plate. In A. Bensoussan and J.L. Lions, editors, Analysis and Optimization of Systems, volume 111 of Lecture Notes in Control and Information Sciences, pages 519-530. Springer-Verlag, Heidelberg, 1988. 